\title{
Software Release Planning
}

\author{
Xavier Franch \\ Group of Software and Service Engineering \\ Universitat Politècnica de Catalunya \\ Barcelona, Spain \\ +93 4137891 \\ franch@essi.upc.edu
}

\author{
Guenther Ruhe \\ Software Engineering Decision Support Laboratory \\ University of Calgary \\ Calgary, Alberta, Canada \\ +1 (403) 2207692 \\ ruhe@ucalgary.ca
}

\begin{abstract}
One of the most critical activities in software product development is the decisional process that assigns features to subsequent releases under technical, resource, risk, and budget constraints. This decision-centric process is referred to as software release planning (SRP). This briefing will expose a state of the art on SRP. A survey of the most relevant approaches will be presented. Emphasis will be made on their applicability (concerning e.g. type of development process - being more predictive versus more adaptive, type of system - commercial, open source product or mobile app), tool support and degree of validation in industry. One of these approaches, EVOLVE, will be analysed in detail.
\end{abstract}

\section{CCS Concepts}

- Software and its engineering $\rightarrow$ Software creation and management $\rightarrow$ Software post-development issues $\rightarrow$ Software evolution.

\section{Keywords}

Software release planning; software evolution.

\section{INTRODUCTION}

One of the most critical activities in software product development is the decisional process that assigns features to subsequent releases under technical, resource, risk, and budget constraints. This decision-centric process is referred to as software release planning (SRP) [1]. It is connected to, and has inspired, various types of research (related to architecture, technical debt, etc.) and is of key importance for the success of software products (continuous delivery, DevOps).

This briefing will expose a state of the art overview on SRP. A survey of the most relevant approaches will be presented. Emphasis will be made on their applicability (concerning e.g. type of development process - being more predictive versus more adaptive, type of system - commercial, open source product or mobile app), tool support and degree of validation in industry. One of these approaches, EVOLVE, will be analysed in more detail.

The briefing is addressed to a wide audience. For researchers, an updated state of the art will be exposed, a particular method will be explored in depth, and the presentation will rely on scientific grounds. For practitioners, the practical dimension of SRP will be

Permission to make digital or hard copies of all or part of this work for personal or classroom use is granted without fee provided that copies are not made or distributed for profit or commercial advantage and that copies bear this notice and the full citation on the first page. Copyrights for thirdparty components of this work must be honored. For all other uses, contact the Owner/Author.

Copyright is held by the owner/author(s).

ICSE ' 16 Companion, May 14-22, 2016, Austin, TX, USA

ACM 978-1-4503-4205-6/16/05.

http://dx.doi.org/10.1145/2889160.2891051 presented. For educators, the briefing will provide the basis for developing course material.

\section{PROPOSAL IN DETAIL}

\subsection{Description of the Topic}

SRP is a complex (NP-hard [2]) decision-centric problem with comprehensive information and knowledge needs. A release is a major (new or upgraded) version of an evolving product characterized by a collection of (new, corrected or modified) features. Good SRP is a mandatory part of incremental and iterative software development. Release decisions address the questions about the functionality (what?), time (when?) and quality (how good?) of product releases.

There are many aspects of SRP that may be subject of analysis. Some refer to scientific topics, like the kind of models and techniques that may be applied or the characterization of inputs and outputs to the process. Others refer to more practical issues, like the extent to what model-based approaches are used compared to judgment-based ones, or the existence of tool support. All these aspects will be considered in the briefing.

\subsection{Interest for SE Community}

All types of release decisions are part of software product management, the discipline of governing a software product from its inception to the market until it is closed down [3]. One of the Standish reports [4] stated that almost two thirds of the features offered are (almost) never used. This not only is a waste of development effort, but also increases maintenance cost and impact usability of the software products.

A recent survey on challenges in product management revealed that half of the product managers consider "the process" the biggest challenge [5]. The survey included almost 900 respondents from around the globe and from a wide variety of industries and company size.

Several research programmes are funding projects related to SRP. We may mention the EU H2020 SUPERSEDE project (www.supersede.eu; 2015-2018) which plans to integrate SRP into a Monitor-Analyse-Decide-Enact loop for the evolution of adaptive systems. Companies like Siemens and Atos act as industrial partners of the project with interest in domains like smart cities and intensive data streaming applications.

It is worth to mention that the topic of the technical briefing is in direct connection with two of the topics listed in the ICSE 2016 call for technical papers: "software evolution and maintenance" and "agile software development".

\subsection{Goal and Objectives}

The overall goal of the technical briefing can be stated as: having an overview of the state of the art in SRP. This goal is broken into the following objectives: 
- Understanding the problem of SRP and the critical success factors for its application.

- Having a broad view of SRP strategies proposed in the literature and clarify their similarities and differences.

- Learning the limitations and applicability conditions of such approaches.

- Analyzing in more depth one particular approach in order to deepen into the problem.

- Provide insight about various extensions of the SRP problem (e.g., theme-based SRP [6], consideration of quality [7]), technical debt [8] or release readiness [9].

- Understanding the role and importance of real-time data analytics and its usage for re-planning [10].

- Recognizing the increasing importance of putting end-users in the loop through feedback gathering [11].

- Get an updated view of the reported state of adoption of such approaches in industry.

\subsection{Overview of the Structure}

The technical briefing is organized with a structure that matches the objectives identified above, organized in 4 parts:

PART I. PRELIMINARIES (15 minutes)

- Introduction of attendees (5 minutes)

- Main concepts (5 minutes)

- A motivating example (5 minutes)

PART II. STATE OF THE ART (35 minutes)

- Information needs (10 minutes)

- Main approaches found in the literature (15 minutes)

- Comparative analysis and consolidation (10 minutes)

PART III. THE EVOLVE APPROACH (30 minutes)

- EVOLVE and related methods (20 minutes)

- The ReleasePlanner tool (10 minutes)

PART IV. CONCLUSIONS (10 minutes)

- Summary (5 minutes)

- Questions (5 minutes)

\subsection{Links to Material}

The main reference for the technical briefing will be Ruhe's text book on release planning methods and tools [1]. Several papers will be keystone for the tutorial, remarkably the systematic review conducted by Svahnberg et al. on strategic release planning models [12] and the recent article on analytical product release planning [10]. The state of the art and the practice on SRP made in the H2020
SUPERSEDE project (www.supersede.eu; to be available as public deliverable for the time of ICSE 2016) will be also a fundamental asset for the briefing.

As for the tool support, we will use during the briefing ReleasePlanner ${ }^{\mathrm{TM} 1}$ 2.0. ReleasePlanner is a proprietary tool that integrates the experience and intuition of the human experts with the computational strength of optimization algorithms. The tool has been grown out of academic research. A free trial for the tool is available at http://rp2.releaseplanner.com/edi/trial_accounts/new. The tool will be used during the briefing for some short hands-on exercise.

\section{ACKNOWLEDGMENTS}

This work is a result of the SUPERSEDE project, funded by the European Union's Information and Communication Technologies Programme (H2020) under grant agreement no 644018.

\section{REFERENCES}

[1] Ruhe, G. 2010. Product Release Planning: Methods, Tools and Applications. CRC Press.

[2] Bagnall, A., Rayward-Smith, V., Whittley, I. 2001. The Next Release Problem. IST 43(14).

[3] Ebert, C., Brinkkemper, S. 2014. "Software Product Management-An Industry Evaluation”. JSS 95.

[4] Standish Group Report, presented at XP2002.

[5] 280 Group. "Challenges in Product Management - Survey Results". Available at: http:/go.280group.com/challenges-inproduct-management-survey-results.pdf, last accessed $17 / 11 / 15$

[6] Karim, M.R., Ruhe, G. 2014. "Bi-objective Genetic Search for Release Planning in Support of Themes". SSBSE 2014.

[7] Felderer, M., Beer, A., Ho, J., Ruhe, G. 2014. "Industrial Evaluation of the Impact of Quality-driven Release Planning”. ESEM 2014.

[8] Ho, J., Ruhe, G. 2014. "When-to-release Planning in consideration of Technical Debt”. MTD@ICSME 2014.

[9] Alam, S., Shahnewaz, S.M., Pfahl, D., Ruhe, G. 2014. "Monitoring Bottlenecks in Achieving Release Readiness - A Retrospective Case Study across Ten OSS Projects". ESEM 2014.

[10] Nayebi, M., Ruhe, G. 2015. Analytical Product Release Planning. In: The Art and Science of Analyzing Software Data (C. Bird, T. Menzies, T. Zimmermann, eds.), Morgan Kaufmann, 2015.

[11] Franch, X., Perini, A., Seyff, N. 2013. "Enabling Informed Decision Making Through Mobile Technologies: A Challenge for Software Engineering”. ICSOFT 2013.

[12] Svahnberg, M., Gorschek, T., Feldt, R., Torkar, R., Bin Saleem, S., Usman Shafique, M. 2010. "A Systematic Review on Strategic Release Planning Models”. IST 52(3).

\footnotetext{
${ }^{1} \mathrm{http}: / / \mathrm{www}$. expertdecisions.com/
} 